\title{
SPATIAL INTERPOLATION OF DAILY HUMIDITY USING NATURAL NEIGHBOURS OVER MOUNTAIN AREAS IN SOUTH EASTERN AUSTRALIA
}

\author{
K.S. KIM, R.M. BERESFORD and W.R. HENSHALL \\ The Horticulture and Food Research Institute of New Zealand Limited, \\ Mt Albert Research Centre, Private Bag 92169, Auckland, New Zealand \\ Corresponding author: kkim@hortresearch.co.nz
}

\begin{abstract}
Natural neighbour interpolation was investigated to estimate daily humidity at specific sites in a mountain area. The Global Summary of Day (GSOD) dataset was used to obtain weather data in mountain areas in south eastern Australia. Eighteen weather stations were selected as validation sites. Dew point temperature was estimated from January to December 2007. When the inverse distance weight method was used without adjusting the elevation difference between stations, accuracy of virtual dew point temperature was poor with a mean absolute error (MAE) of $3.6^{\circ} \mathrm{C}$. When natural neighbour interpolation was used, the MAE for dew point temperature was $2.1^{\circ} \mathrm{C}$ with altitude adjustment. Furthermore, application of wet adiabatic lapse rate $\left(-0.004^{\circ} \mathrm{C} / \mathrm{m}\right)$ for altitude adjustment reduced the MAE to $1.3^{\circ} \mathrm{C}$. These results will be used to improve the accuracy of weather estimates in areas with complex terrain, in order to implement crop disease predictions using risk models.

Keywords: disease prediction, weather data, spatial interpolation, natural neighbour.
\end{abstract}

\section{INTRODUCTION}

Humidity is an important driving variable for many plant pathological and plant physiological processes (Wilks \& Shen 1991; Rawson et al. 1977). Simulation of such processes depends on accurate humidity data. However, measurements of humidity are relatively scarce compared with those of temperature and precipitation.

Humidity can be estimated using existing weather stations located near a site of interest. Spatial interpolation has been used to estimate various weather variables using neighbouring stations (Snell et al. 2000; Chung et al. 2006). Jarvis \& Stuart (2001) compared spatial interpolation methods for estimating daily maximum and minimum temperature.

When weather variables are estimated using spatial interpolation, it is important to take into account terrain conditions between weather stations. A lapse rate correction is often used in spatial interpolation of temperature to take elevation difference between weather stations into account (Chung et al. 2006). For example, Snell et al. (2000) interpolated daily maximum temperature using environmental lapse rate $\left(0.0065^{\circ} \mathrm{C} / \mathrm{m}\right)$, which is the change of temperature with altitude of an air parcel.

It is likely that spatial interpolation of humidity is affected by the terrain surrounding a point of interest. Terrain effect on humidity can be adjusted using the wet adiabatic lapse rate that applies to a saturated air parcel ascending within the atmosphere. The objectives of the present study were to determine the accuracy of interpolated humidity estimates using the natural neighbour method and to apply altitude adjustment to spatial interpolation in order to increase their accuracy. 


\section{MATERIALS AND METHODS}

The US National Climate Data Center (NCDC) Global Surface Summary of the Day (GSOD) data were used in this study (Lott 1998). The GSOD database includes air temperature, dew point temperature, precipitation and other daily weather variables. The area selected for the study was southeast New South Wales and northeast Victoria in Australia, because there are a large number of weather stations available in the GSOD database for this region (Fig. 1). Stations with altitude greater than $400 \mathrm{~m}$ above sea level were selected as validation sites to assess effects on terrain in spatial interpolation of humidity. Weather data were collected at neighbour weather stations as well as validation sites from January to December in 2007.

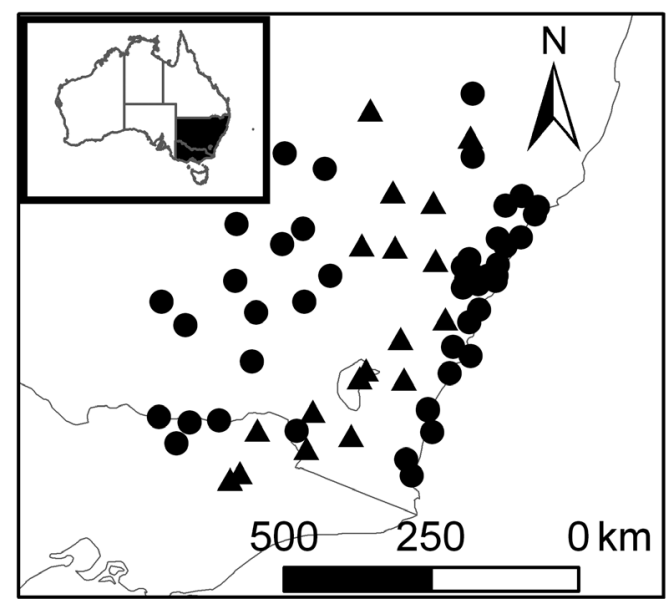

FIGURE 1: The 66 weather stations in southeast New South Wales and northeast Victoria included in the present study. Circles and triangles represent neighbour and validation sites, respectively.

Daily dew point temperature was estimated using the natural neighbour method. In the natural neighbour algorithm, distance and bearing of weather stations are used to select neighbours of a site of interest. In the process, the geometrical technique known as Voronoi tessellation is used to find areas $\kappa$ that surround the point of interest (Sibson 1980). To interpolate dew point temperature $V$ from neighbour stations, $M$, Shepard's and Sibson's methods were used as follows (Sibson 1980; Snell et al. 2000; Kim et al. 2007):

$$
V=\sum_{i}\left[M_{i} \cdot\left(\frac{d_{i}^{2}}{\sum_{k} d_{k}^{2}}\right)^{-1}\right] / \sum_{i}\left(\frac{d_{i}^{2}}{\sum_{k} d_{k}^{2}}\right)^{-1}
$$

and

$$
V=\sum_{i}\left[M_{i} \cdot\left(\frac{\kappa_{i}}{\sum_{k} \kappa_{k}}\right)\right] / \sum_{i}\left(\frac{\kappa_{i}}{\sum_{k} \kappa_{k}}\right)
$$

where $d_{i}$ and $\kappa_{i}$ are distance and area between a validation site and neighbour weather station, i, respectively. Mean absolute error (MAE) was calculated to analyse errors in 
spatial interpolation. Wilcoxon's signed rank test was also performed to compare the MAE of Shepard's and Sibson's methods (Snell et al. 2000).

To take into account the likely gradient of humidity between two sites with different elevations, the environmental lapse rate $(\Gamma)$ was applied to measurements at a neighbour station as follows:

$$
M_{i}^{\prime}=M_{i}-\Gamma\left(\beta_{i}-\beta\right)
$$

where $\Gamma=0.0065^{\circ} \mathrm{C} / \mathrm{m}$ and $\beta=$ the altitude of a weather station. The wet adiabatic lapse rate $\Gamma^{\prime}\left(0.004^{\circ} \mathrm{C} / \mathrm{m}\right)$ was also used to apply altitude adjustment between validation and neighbour weather stations. A computer programme written in $\mathrm{C}++$ was developed to implement interpolation of dew point temperature using the above procedures.

\section{RESULTS}

When Shepard's method was used to interpolate dew point temperature without applying any lapse rate adjustment for altitude between weather stations (Fig. 2a), the mean absolute error (MAE) of interpolated dew point temperature was $3.6^{\circ} \mathrm{C}$ $\left(\mathrm{R}^{2}=0.83\right)$. MAE reduced to $2.3^{\circ} \mathrm{C}$ when altitude adjustment using the environmental lapse rate was applied to Shepard's method (Fig. 2b). MAE dropped further to about $2.1^{\circ} \mathrm{C}$ when Sibson's method was used with the environmental lapse rate for altitude adjustment (Fig. 2c).

Interpolated dew point temperature was mostly underestimated when the environmental lapse rate was used to adjust the dew point for the altitude difference between weather stations (Fig. 2b, c). However, this underestimation error reduced to $1.3^{\circ} \mathrm{C}\left(\mathrm{R}^{2}=0.90\right)$ when the wet adiabatic lapse rate was used instead of the environmental lapse rate with Sibson's method (Fig. 2d). In addition, Sibson's method had a significantly lower MAE than Shepard's method $(\mathrm{P}=0.000)$.
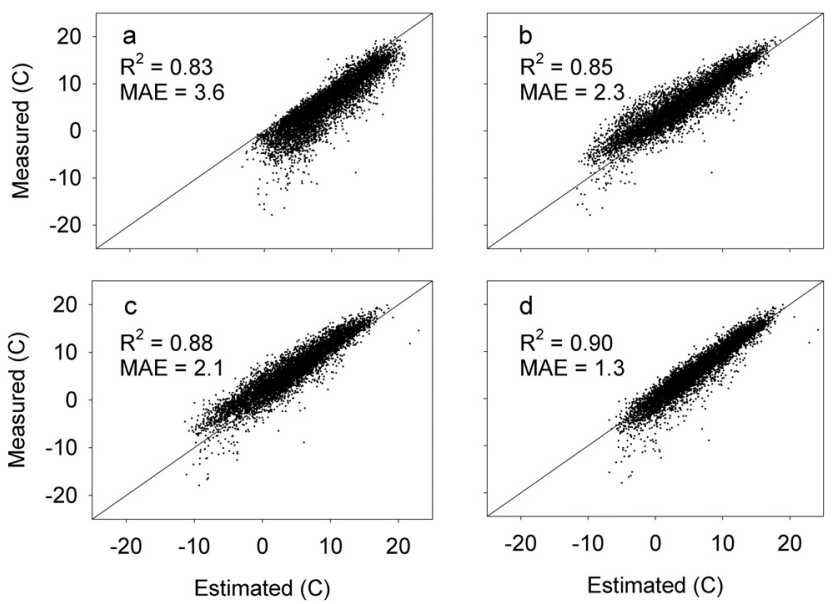

FIGURE 2: Daily dew point temperature measurements and estimates using (a) Shepard's method without applying altitude adjustment between weather stations, (b) Shepard's method with altitude adjustment using environmental lapse rate $\left(0.0065^{\circ} \mathrm{C} / \mathrm{m}\right)$, (c) Sibson's method with the altitude adjustment as (b), and (d) Sibson's method using the wet adiabatic lapse rate $\left(0.004^{\circ} \mathrm{C} / \mathrm{m}\right)$ for altitude adjustment. MAE is mean absolute error of interpolated dew point temperature. 


\section{DISCUSSION}

These results showed that more accurate spatial interpolation was achieved when daily dew point temperature was corrected for altitude differences between weather stations. The wet adiabatic lapse rate gave better correction than the environmental lapse rate, as could be expected because the dew point temperature better reflects the physical property of saturated air parcels. This suggests that hourly humidity estimation using spatial interpolation can benefit from the approach presented here.

Kimball et al. (1997) reported an empirical model to estimate daily humidity using on-site measurements of daily minimum temperature. The standard error of their model ranged from $2.1^{\circ} \mathrm{C}$ to $5.3^{\circ} \mathrm{C}$. In contrast, Sibson's method with the wet adiabatic lapse rate for altitude adjustment had the standard error of $1.8^{\circ} \mathrm{C}$.

Leaf wetness duration (LWD) has been used as a key input variable in disease warning systems (Kim et al. 2007). Wilks \& Shen (1991) reported that LWD could be estimated accurately using humidity. Therefore, the approach presented here could lead to wide implementation of models to predict the risk of a plant disease at sites where no measurement of LWD or humidity is available.

In this study, the wet adiabatic lapse rate was assumed to be constant $\left(0.004^{\circ} \mathrm{C} / \mathrm{m}\right)$. However, the wet adiabatic lapse rate is variable under different temperature and pressure conditions. In a future study, the wet adiabatic lapse rate could be parameterised using temperature and pressure and used in an interpolation method to give an even better estimation of atmospheric humidity.

\section{ACKNOWLEDGEMENTS}

This study was carried out with financial support from the HortResearch Capability Fund.

\section{REFERENCES}

Chung U, Seo HH, Hwang KH, Hwang BS, Choi J, Lee JT, Yun JI 2006. Minimum temperature mapping over complex terrain by estimating cold air accumulation potential. Agricultural and Forest Meteorology 137:15-24.

Jarvis CH, Stuart N 2001. A comparison among strategies for interpolating maximum and minimum daily air temperatures. Part II: The interaction between number of guiding variables and the type of interpolation method. Journal of Applied Meteorology 40:1075-1084.

Kim KS, Beresford RM, Henshall WR 2007. Prediction of disease risk using site-specific estimates of weather variables. New Zealand Plant Protection 60: 128-132.

Kimball JS, Running SW, Nemani R 1997. An improved method for estimating surface humidity from daily minimum temperature. Agricultural and Forest Meteorology 85: 87-98.

Lott N 1998. Global surface summary of day (http://lwf.ncdc.noaa.gov/cgi-bin/res40. pl?page $=$ gsod.html), National Climatic Data Center, Asheville, N.C.

Rawson HM, Begg JE, Woodward RG 1977. The effect of atmospheric humidity on photosynthesis, transpiration and water use efficiency of leaves of several plant species. Planta 134:5-10.

Sibson R 1980. A vector identity for the dirichlet tessellation. Mathematical Proceedings of the Cambridge Philosophical Society 87: 151-155.

Snell SE, Gopal S, Kaufmann RK 2000. Spatial interpolation of surface air temperature using artificial neural networks: evaluating their use for downscaling GCMS Journal of Climate 13: 886-895.

Wilks DS, Shen KW 1991. Threshold relative humidity duration forecasts for plant disease prediction. Journal of Applied Meteorology 30: 463-477. 